\title{
Justification of the air separation curve
}

\author{
Rinat Sharapov* \\ Moscow State University of Civil Engineering, Yaroslavskoe shosse, 26, Moscow, 129337, Russia
}

\begin{abstract}
The article considers a method for increasing the production efficiency of fine powders. It is shown that modern air separators do not have high efficiency. A method for increasing the efficiency of air separators is proposed. It is noted that the main characteristic of the separation process is the separation curve, with the help of which a dispersed composition of fine and coarse products can be found. The calculation is made taking into account the turbulent diffusion of separation air in the casing of the air separator, and also the constructive features of the separator in question. The separation function is proposed taking into account the proposed technical solution.
\end{abstract}

\section{Introduction}

The production of fine powders is a very urgent task. This is explained by the fact that such powders allow intensify chemical, heat and mass exchange, thermal heterogeneous processes. In this case, after traditional methods of grinding, ready-mixed powders can contain particles that do not always satisfy the requirements of effective subsequent technological processes [1-3]. To solve these problems, air separators of various designs are widely used [4-10].

However, even modern air separators do not have a high separation efficiency. This parameter is 55-65\%. To increase this parameter, we suggest that the separating air circulating in the system be thrown into aspiration. The missing air must be added directly to the separating air inlet into the separator. This measure should improve the efficiency of the grinding unit as a whole.

\section{Teoretical}

The main characteristic of the cement separation process is the separation curve $\varphi(d)$, with which it is possible to find the dispersed composition of fine and coarse products, and also to calculate the efficiency of the separator. In centrifugal air separators, an ideal separation of the material into a fine $\left(d<d_{b}\right)$ and coarse $\left(d>d_{b}\right)$ fraction is not possible, since all particles due to intense turbulent placement are distributed throughout the cross section of the separation zone.

This leads to the fact that some of the fine particles get into the tails, and a number of large particles into a thin product. For this reason, the separator separation curve can not be

\footnotetext{
* Corresponding author: rinat.sharapoff@gmail.com
} 
derived within the framework of the deterministic approach, i.e. on the basis of only the equations of particle motion in the averaged velocity field of separation air. When deriving the separation curve, it is necessary to take into account the random perturbations of the particle motion caused by turbulent pulsations of the separating air carrier stream and mutual collisions of particles:

$$
\nabla\left(Z \bar{v}-D_{t p} \nabla Z\right)=0
$$

where $\nabla$ is the differential nabla operator; $Z$ - concentration of particles, $\mathrm{kgpm}^{3} ; \bar{v}$ is the velocity field of the aerosol liquid; $D_{t p}$ is the coefficient of turbulent diffusion of particles, which characterizes the square of the average deviation of the particles from their deterministic trajectories per unit time. The value of $D_{t p}$ can be estimated from the empirical formula [11]:

$$
D_{t p}=\frac{v_{t}}{1+0.286 \mathrm{Stk}}
$$

where $v_{t}$ is the kinematic coefficient of the turbulent viscosity of the air; Stk is the Stokes criterion:

$$
\mathrm{Stk}=\frac{U_{r}\left(R_{1}\right) \tau}{2 R_{1}},
$$

According to the hypothesis of A.M. Obukhov and A.N. Kolmogorov [11], the coefficient of turbulent diffusion of air is determined by the formula:

$$
v_{t}=a \varepsilon^{1 / 3} l^{4 / 3}, \mathrm{~m}^{2} \mathrm{ps},
$$

where $a$ is the empirical coefficient; $\varepsilon$ - energy dissipated per 1 second in $1 \mathrm{~kg}$ of air, Wpkg; $l$ is the linear scale of the turbulence, $\mathrm{m}$. The value of $\varepsilon$ is proportional to the hydraulic resistance of the separator $\Delta p_{\mathrm{c}}$ and can be estimated using the relation:

$$
\varepsilon=\frac{\Delta p_{c} v_{r}\left(R_{1}\right)}{\rho R_{1}} .
$$

As the scale of turbulence, the internal radius of the separation zone can be chosen:

$$
l=R_{1} .
$$

Substituting relations (5) and (6) into formula (4), we obtain:

$$
v_{t}=a \sqrt[3]{\frac{\Delta p_{c} R_{1}^{2} G}{2 \pi H \rho^{2}}} .
$$

Equation (7) can be solved under appropriate boundary conditions in conjunction with the equations of aerodynamics only by numerical methods. Based on the results of calculations, relative fractions (partial removals) of individual fractions of particles into a thin product can be found. The separation curves are obtained by approximating these outliers by analytical expressions satisfying the following requirements:

$$
\begin{aligned}
& \varphi(d) \rightarrow 1 \text { if } d \rightarrow 0, \\
& \varphi(d) \rightarrow 1 \text { if } d \rightarrow \infty,
\end{aligned}
$$




$$
\varphi\left(d_{b}\right)=0.5
$$

One such expression is the Molerus' equation [12]:

$$
\varphi(\tilde{d})=\frac{1}{1+\tilde{d} \exp \left(-\frac{A}{2}(1-\tilde{d})\right)},
$$

where $\tilde{d}=d / d_{b} ; A$ is the stochastic parameter equal to the Peclet diffusion criterion for the disperse phase [13]:

$$
A=\frac{2 R_{1} v_{r}\left(R_{1}\right)}{D_{\mathrm{tp}}},
$$

Parameter $A$ characterizes the degree of influence of random factors on the ordered process of separation under the influence of aerodynamic drag force and centrifugal force. With decreasing $D$, the parameter $A$ increases, and for $A \geq 16$ the separation curve approaches the ideal curve [12].

With parameter $A$, the sharpness of the separation is related $\xi=d_{0.75} / d_{0.25}$, where $d_{0.75}$ and $d_{0.25}$ are the particle sizes, which are converted by the separator into a thin product by 75 and $25 \%$. For an ideal classification, $\xi=1$ (Fig. 1).

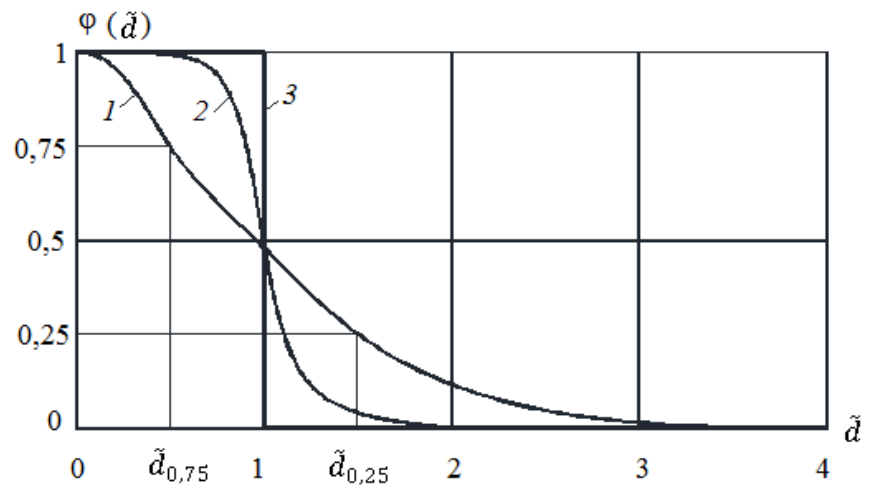

Fig. 1. Influence of the value of the stochastic parameter $A$ on the shape of the separation curve:

$$
1-A=3, \xi=0.36 ; 2-A=15, \xi=0.6 ; 3-A_{-}^{-} \infty, \xi=1 \text { (ideal classification) }
$$

The shape of the separation curve, and therefore the performance of the separator, is also affected by the value of $d_{b}$.

At present, there is no theoretical predictor for the value of the parameter $A$, so its value should be determined experimentally from the experimental values of the distribution function $\varphi(d)$ for various $d$.

From the equation of the material balance of a narrow fraction of the particle size $d$ :

$$
G_{m} f_{m}(d) \varphi(d)=G_{f} f_{f}(d)
$$

follows:

$$
\varphi(d)=\frac{G_{f}}{G_{m}} \cdot \frac{f_{f}(d)}{f_{m}(d)}=\frac{1}{k_{c}} \cdot \frac{R_{f}(d+\Delta d)-R_{f}(d)}{R_{m}(d+\Delta d)-R_{m}(d)},
$$


where $G_{m}$ and $G_{f}$ are the mass flows of the starting material and the thin product; $k_{c}$ is the multiplicity of the circulation, $f_{m}(d), f_{f}(d)$ are the differential distribution functions; $R_{m}(d)$, $R_{f}(d)$ are the integral functions of the distribution of the starting material and the thin product, which are determined from the results of laser granulometry.

The separation function (11) by logarithm and simple transformations can be reduced to a linear form:

$$
y=S x
$$

where

$$
\begin{array}{r}
y=2 \ln \left(\frac{\varphi(d) \cdot \tilde{d}}{1-\varphi(d)}\right), \\
x=1-\tilde{d}, \\
\tilde{d}=d / d_{b} .
\end{array}
$$

Having determined the values of $\varphi\left(d_{i}\right), i=1,2, \ldots n$, using the formula (14), the value of the parameter $A$ can be found by the method of least squares:

$$
A=\frac{\sum_{i=1}^{n} y_{i} \cdot x_{i}}{\sum_{i=1}^{n} x_{i}^{2}} .
$$

Parameter $A$ values were determined under production conditions at different fresh air flow rates. As a result of processing the obtained data, the following approximating dependence of the stochastic parameter on the mass fraction of fresh air $K$ in the entire separation air stream was obtained:

$$
A=-31.2 K^{2}+44.34 K-1.14 .
$$

The plot of the dependence (20) is shown in Fig. 2, from which it follows that an increase in the fraction of fresh air in separation air leads to an increase in the stochastic parameter and, as shown in Fig. 1, to improve the efficiency of separation.

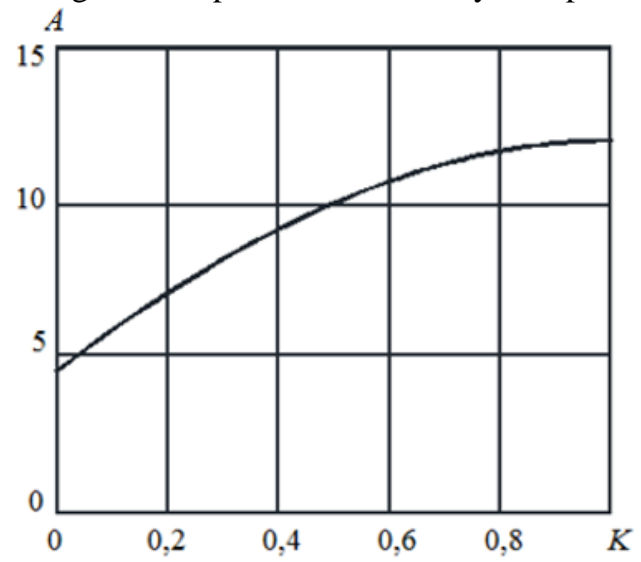

Fig. 2. Dependence of the stochastic parameter $A$ on the mass fraction of fresh air in the separation air 


\section{Conclusion}

An increase in the parameter $A$ with an increase in the fraction of fresh air $K$ is explained by a decrease in the temperature of the separation air, which leads to a decrease in its volumetric flow rate and the velocity of the gas-material flow. As a result, the level of turbulence of the gas material medium decreases, the coefficient of turbulent diffusion of the dispersed phase decreases, which leads to an increase in the stochastic parameter $A$.

\section{References}

1. Y. M. Butt, V. V. Timashov, Portlandcement, (1974).

2. V. A. Perov, E. E. Andreev, L. F. Bilenko, Crushing, grinding and screening of minerals (1990).

3. R.R. Sharapov, P.D. Kapyrin S.Y. Lozovaya, V.V. Yadykina, A.M. Agarkov, MATEC Web of Conferences 5. "5th International Scientific Conference "Integration, Partnership and Innovation in Construction Science and Education", IPICSE 2016". 03007. (2016).

4. W. H. Duda, Cement-data-book. Band 1 (Internationale Verfahrenstechniken der Zementindustrie, Wiesbaden, Berlin: Bauverlag) (1985).

5. T. Furukawa, E. Onuma, T. Misaka. Int. Symp. on powder technology. (1981).

6. E. Hanke, Wor. Cem. 17. 3. (1986).

7. A. Subeggve, ZKG Int. 2. (2004).

8. R. Ironman, RP 84. 4. (1980).

9. Y. Reinchardt, Wor. Cem. March. (2008).

10. V.I. Telichenko, R.R. Sharapov, S.Y. Lozovaya, V.I. Skel, MATEC Web of Conferences 5. "5th International Scientific Conference "Integration, Partnership and Innovation in Construction Science and Education", IPICSE 2016". 04040. (2016).

11. E. P. Mednikov, Turbulent transference and aerosol deposition, (1980).

12. V. E. Mizonov, S. G. Ushakov, Aerodynamic classification of powders (1989).

13. R.R. Sharapov, I.V. Shrubchenko, Rinat R. Sharapov, A. M. Agarkov IJAER 10. 12. (2015). 